\title{
Safe sedation practices among gastroenterology registrars: do we need more training?
}

\author{
Aruchuna Mohanaruban, ${ }^{1}$ Kathleen Bryce, ${ }^{2}$ Archchana Radhakrishnan, ${ }^{3}$ \\ Joseph Gallaher, ${ }^{4}$ Gavin Johnson ${ }^{5}$
}

1 Department of Gastroenterology, St Mary's Hospital, London, UK

2Princess Alexandra Hospital, Harlow, UK

${ }^{3}$ St Mary's Hospital, London, UK

${ }^{4}$ Broomfield Hospital,

Chelmsford, Essex, UK

${ }^{5}$ University College Hospital, London, UK

\section{Correspondence to}

Dr Aruchuna Mohanaruban, Department of Gastroenterology, St Mary's Hospital, London W2 1NY, UK:

Aruchuna@gmail.com

Received 23 December 2014 Revised 21 March 2015 Accepted 23 March 2015 Published Online First 17 April 2015

\section{CrossMark}

\author{
To cite: Mohanaruban $A$, \\ Bryce K, Radhakrishnan A, \\ et al. Frontline \\ Gastroenterology \\ 2015:6:223-228.
}

\begin{abstract}
Endoscopy training is a central component of gastroenterology training for the vast majority of UK trainees, and integral to this is the practice of safe sedation. The majority of endoscopic procedures are performed with the patient under conscious sedation with a benzodiazepine, often combined with an opioid. Little data exists on the practice of sedation among gastroenterology trainees, including their degree of knowledge of the common sedation agents used and their actions. Using both an online and paper-based questionnaire, we surveyed current gastroenterology speciality trainees (ST) in the UK and received 78 responses giving a response rate of $10 \%$. Fifty-one per cent of the trainees did not receive structured training in safe sedation, despite national guidelines advising this to be an essential part of the training programme, and $92 \%$ felt a structured sedation course would be beneficial. We also identified some gaps in trainees' knowledge of the action of sedation agents. We propose that a formal training session in sedation or an e-learning module could be incorporated as part of a deanery or trust induction for gastroenterology trainees and kept under regular review.
\end{abstract}

\section{INTRODUCTION}

The increasing volume of therapeutic endoscopy coupled with more procedures conducted on an ever-ageing population has led to a greater emphasis on performing safe sedation in the endoscopy department. The majority of endoscopic procedures are performed with the patient under conscious sedation. ${ }^{1}$ The aim is to minimise anxiety and discomfort in order to complete the procedure safely and successfully. ${ }^{1}$ The most commonly used sedation agents are opioids (usually fentanyl) and midazolam, which are both given intravenously. ${ }^{2}$ Patients may also opt to have the procedure performed unsedated or use suitable alternatives such as Xylocaine throat spray or nitrogen oxide (Entonox), although the latter is used infrequently in the UK. ${ }^{3}$

The Department of Health sets out a list of 'never events', which are defined as serious, but preventable, patient safety incidents. ${ }^{4}$ These include over sedation with midazolam and failure to monitor or to respond to oxygen saturations; both are not uncommon events.

The British Society of Gastroenterology (BSG) published guidelines for the administration of safe sedation in 2003, which clearly stated that 'safety will only be optimised if practitioners use defined methods of sedation for which they have received formal training'. Following on from this in 2004, the National Confidential Enquiry into Patient Outcome and Death (NCEPOD) highlighted that only $35 \%$ of endoscopists surveyed were known to have attended a course on safe sedation. ${ }^{5}$ This report recommended that all those responsible for the administration of sedation in the endoscopy department should receive formal training, and clear protocols for the administration of sedation should be made available and implemented.

Sedation protocols and auditing their use as well as their reversal agents are quality and safety indicators as set out by The Joint Advisory Group (JAG) on gastrointestinal (GI) endoscopy who regulate gastroenterology training in the UK. ${ }^{6}$ In 2004, JAG developed the Global Rating Scale (GRS), a patient-centred web-based self-assessment tool, to evaluate and improve all aspects of endoscopy. ${ }^{7}$ Patient 
safety and comfort is assessed using this tool under the domain of clinical quality and integral to this is the use of safe sedation. ${ }^{8}$

Despite these frameworks and recommendations set out by both NCEPOD and JAG, there have been very few studies to date that survey the current practice and level of training among gastroenterology trainees. Therefore, the primary aim of this study was to establish whether gastroenterology trainees had received any formal training in administering safe sedation in the endoscopy department and explore how this training was delivered. The secondary aim was to determine trainees' current practices in delivering the common sedation agents, including midazolam and fentanyl.

\section{METHODS}

A 19-question survey (table 1) was developed, which was distributed at a gastroenterology training course held in London and also made available online using the web-based Survey Monkey. ${ }^{9}$ A direct link for the survey was e-mailed to all trainee members of the BSG. Data was collected from December 2013 to June 2014. The lines of enquiry were the trainee grade, deanery, whether formal training in sedation had been received, and, if so, in what format had this training been delivered. Trainees were asked not to consider the 'Basic Skills in Upper GI Endoscopy' when answering this question as this course is a mandatory requirement by JAG, and we wanted to elicit whether additional focused training in sedation had

Table 1 Full list of questions asked in the national training survey

Question

1.1. What is your current level of training?

1.2. Where do you work? Deanery

2.1. Have you received formal training in sedation for endoscopy?

2.2. If yes, please tick all that apply

3. Do you think receiving an introductory course or lecture in sedation at the beginning of endoscopy training would be useful?

4. Are you aware of a protocol in your endoscopy department for the administration of sedation?

5. Are you aware of where reversal agents for sedation are located in your department?

6. Which opioid is used as first-line in your department?

7. How often do you use an opioid when performing diagnostic upper GI endoscopy?

8. Are the majority of diagnostic upper GI endoscopies that you perform sedated or unsedated (throat spray only)?

9. Which do you choose to administer first when performing a diagnostic colonoscopy?

10. On average, what dose of midazolam do you administer?

10.1 For diagnostic upper $\mathrm{Gl}$ endoscopy?

10.2 For diagnostic colonoscopy?

11. What is the maximum dose of midazolam that you would feel comfortable giving?

12. What is the maximum dose of fentanyl that you would feel comfortable giving?

13. What initial dose of midazolam do you use for an elderly patient undergoing

13.1 Upper GI endoscopy

13.2 Colonoscopy

14. How long does midazolam take to act?

15. When is the peak effect of midazolam?

16. How long does fentanyl take to act?

17. When is the peak effect of fentanyl?

18. What is the reversal agent for midazolam?

19. What is the reversal agent for fentanyl?
Answer format

Tick box answer

ST3/ST4/ST5/ST6/ST7/ST8/Other

Free text box

Yes/No

Tick box answer

A dedicated course in safe sedation (excluding JAG Basic Skills in Upper Gl Endoscopy Course)/Local trust induction/E-learning

Yes/No

Yes/No/Don't know

Yes/No

Tick box answer

Fentanyl/Pethidine/Other

Tick box answer

Always/Very often/Sometimes/Rarely/Never

Sedated/Unsedated

Opioid (fentanyl/pethidine) followed by midazolam/Midazolam followed by opioid (fentanyl/pethidine)/Other regime (please specify)

Free text box_mg

Free text box_mg

Free text box $\_\mu g$

Free text box_mg

Tick box answer

$<1 \mathrm{~min} / 1-2 \mathrm{~min} / 3-5 \mathrm{~min} />5 \mathrm{~min}$

Tick box answer

$<1 \mathrm{~min} / 1-2 \mathrm{~min} / 3-5 \mathrm{~min} />5 \mathrm{~min}$

Tick box answer

$<1 \mathrm{~min} / 1-2 \mathrm{~min} / 3-5 \mathrm{~min} />5 \mathrm{~min}$

Tick box answer

$<1 \mathrm{~min} / 1-2 \mathrm{~min} / 3-5 \mathrm{~min} />5 \mathrm{~min}$

Free text box

Tick box: don't know

Free text box

Tick box: don't know 
been undertaken by the trainees. Trainees were also asked if they were aware of a local protocol for sedation in their hospital, and whether they were aware of where reversal agents for opioids and midazolam were located. The final part of the questionnaire asked trainees to record their current practices of midazolam and opioid administration as well as to undertake a knowledge test on the basic pharmacokinetics and reversal agents of both drugs, respectively. Data was analysed using descriptive statistics with Microsoft Excel. When analysing the data, the trainees were also split into two cohorts, those who had attended formal training in sedation and those who had not received any formal training, to compare and contrast the results from these two groups.

\section{RESULTS}

Seventy-eight specialist registrars in gastroenterology responded to the questionnaire out of 758 BSG trainees nationwide, giving a response rate of $10 \%$. The majority were trainees based in London (51\%) with the rest of England (46\%) also well represented. The remaining $3 \%$ were from Wales, Scotland and Northern Ireland.

Only $49 \%$ of the responding trainees reported having received formal training in sedation for endoscopy, predominantly at local trust inductions $(32 \%)$ or a dedicated course in safe sedation (46\%) (table 2). Less than half of the trainees $(46 \%)$ were aware of their local trust protocols for sedation in endoscopy.

All the trainees surveyed knew the correct reversal agents for the commonly used sedation agents in endoscopy, but 19\% were unsure where reversal agents were kept in their department.

Overall, fentanyl was the first choice of opioid agent used with $70 \%$ preferring to use this drug over pethidine among those who had attended formal sedation courses compared to the non-formally trained cohort where $63 \%$ used fentanyl as their first-line opioid (table 3).

Sedation was found to be used rarely in diagnostic upper GI endoscopy with the majority electing to use Xylocaine throat spray, irrespective of whether the trainee had received formal training in sedation or not. Twenty per cent of the trainees who had received formal training in sedation stated that they performed the majority of upper GI endoscopies using some form of sedation, compared to $26 \%$ in the nonformally trained cohort (table 4).

Table 2 Formal sedation training received

\begin{tabular}{lc}
\hline Delivery of sedation training & Percentage \\
\hline Anaesthetic training on sedation & 9 \\
Dedicated course in safe sedation & 46 \\
e-Learning & 13 \\
Local trust induction & 32 \\
\hline
\end{tabular}

Table 3 First-line opioid used in department

\begin{tabular}{ll}
\hline First-line opioid used & \\
\hline Formal sedation training & \\
Fentanyl & $70 \%(26)$ \\
Pethidine & $30 \%(11)$ \\
Non-formal training sedation training & \\
Fentanyl & $63 \%(22)$ \\
Pethidine & $37 \%(13)$ \\
\hline
\end{tabular}

When trainees were asked how often they used an opioid during upper GI endoscopy, $74 \%$ of formally trained responders opted for 'rarely or never' compared to $71 \%$ in the non-formally trained group (table 5). Furthermore, 5\% in the non-formally trained cohort stated that they used opioids 'very often'.

Ninety-five per cent of trained responders correctly used an opioid followed by midazolam in their sequence of sedation agents when performing colonoscopy. This was $86 \%$ in the non-formally trained group (table 6).

All trainees suggested reasonable average doses for midazolam use in upper GI endoscopy and for diagnostic colonoscopy. The majority of trainees stated appropriate estimates for the maximum safe dose of midazolam and fentanyl and tended to use an initial dose of $1 \mathrm{mg}$ of midazolam for elderly patients undergoing gastroscopy and colonoscopy (table 7 ).

Both trained and untrained cohorts stated reasonable times for the duration of action of fentanyl and midazolam and the peak of their effects. Ninety-one per cent of responders felt that a formal introductory course in sedation would be useful.

\section{DISCUSSION}

A wide cross section of current gastroenterology trainees were surveyed, ranging from speciality trainees ST3 to ST7 representing a wide spectrum of experience. To our knowledge, this is the first nationwide study to investigate gastroenterology trainees' perceptions and the practice of sedation in the endoscopy department. The curriculum for gastroenterology training in the UK recommends that trainees ensure

Table 4 Use of sedation among trainees relative to training status

\begin{tabular}{ll}
\hline Are the majority of OGDs you perform sedated? & \\
\hline Formal training & \\
Yes & $20 \%(8)$ \\
No & $80 \%(30)$ \\
Non-formal training & \\
Yes & $26 \%(10)$ \\
No & $74 \%(28)$ \\
\hline
\end{tabular}


Table 5 How often do you use an opioid when performing diagnostic upper $\mathrm{Gl}$ endoscopy?

\begin{tabular}{lc}
\hline $\begin{array}{l}\text { How often do you use an opioid } \\
\text { for upper Gl endoscopy? }\end{array}$ & Percentage \\
\hline Formal training & 0 \\
$\quad$ Always & 0 \\
Very often & 26 \\
Sometimes & 51 \\
Rarely & 23 \\
Never & \\
Non-formal training & \\
Always & 0 \\
Very often & 5 \\
Sometimes & 24 \\
Rarely & 34 \\
Never & 37 \\
\hline
\end{tabular}

knowledge of the principles and details of safe endoscopy practice and awareness of the pharmacology of the frequently used drugs. ${ }^{10}$ Sedation carries with it a $1 \%$ risk of hypotension, aspiration, desaturation and bradycardia during endoscopy. ${ }^{11}$

Our findings highlight that only $49 \%$ of our cohort of trainees surveyed had received formal sedation training, which compares to the NCEPOD report findings of just $35 \%$ having received formal training in 2004. This suggests that we are still falling short of NCEPOD recommendations that all individuals responsible for administering sedation in endoscopy should receive formal training to administer these agents. Those trainees who had received formal sedation training mostly did so, on a dedicated sedation course. This demonstrates that trainees are currently seeking out their own formal sedation training, which suggests deficiencies in the national training programme and in local departmental inductions. Some sedation training is incorporated into the mandatory JAG training courses; Basic Skills in Upper or Lower GI Endoscopy. However, trainees are advised to book into this course after having had some experience in performing these procedures beforehand. As a result, most trainees will have already administered sedation to patients before attending this course. These are also both 'one-off' courses and the trainee will not benefit from revision of the principles and practice of

Table 6 Order of agents used in colonoscopy

\begin{tabular}{lc}
\hline Which do you administer first when performing colonoscopy? \\
\hline Formal training \\
$\quad$ Opioid followed by midazolam & $95 \%(35)$ \\
Midazolam followed by opioid & $5 \%(2)$ \\
Non-formal training & \\
Opioid followed by midazolam & $86 \%(30)$ \\
Midazolam followed by opioid & $14 \%(5)$ \\
\hline
\end{tabular}

sedation. It is likely that most sedation training comes from the trainee's supervisor during 'training' endoscopy lists; however, there is likely to be insufficient time to discuss the agents given in detail at these times. The vast majority of trainees have indicated that they would value timely formal sedation training with incorporation into local induction.

Most trainees are not aware of a sedation protocol in their department. Lack of consistency of practice within and between endoscopy units can increase uncertainty for the trainee. Differences in practice were reflected in the range of average and maximum doses for sedation agents given by trainees with some trainees reporting being comfortable using up to $10 \mathrm{mg}$ of midazolam or $200 \mu \mathrm{g}$ of fentanyl. The BSG recommends an average dose of no more than $2 \mathrm{mg}$ of midazolam in the case of elderly patients (defined as over the age of 70 years), while in a fit young adult, a dose of $5 \mathrm{mg}$ might be more appropriate. ${ }^{3}$ These guidelines also advise that doses of fentanyl and pethidine should not exceed $100 \mu \mathrm{g}$ and $50 \mathrm{mg}$, respectively, and that a dose reduction of $50 \%$ is required in the elderly population.

Most trainees do not use an opioid or sedation for upper GI endoscopy, but those trainees who had received formal sedation training were more likely, during colonoscopy, to administer an opioid first, before midazolam.

We found generally good trainee understanding of basic pharmacokinetics of sedation agents, but did identify some gaps in trainees' knowledge of the action of sedation agents. Most trainees knew the recommended maximal doses for midazolam and fentanyl and were appropriately cautious with sedation in elderly patients.

There are several limitations to this study. The number of respondents was small compared to the UK total trainee cohort, giving a response rate of $10 \%$. In addition, the majority of trainees surveyed were from England, making it difficult to generalise these results to apply to the whole of the UK. The small number of respondents also means that differences between the 'received training' and 'did not receive training' cohorts, with respect to subsequent sedation practice, cannot reach statistical significance; so, scientifically robust conclusions cannot be drawn.

\section{CONCLUSIONS}

From this study, we have concluded that the majority of trainees would value formal sedation training. We have also identified some gaps in trainees' knowledge of the action of sedation agents. Our findings highlight the need for a better structured training for gastroenterology trainees in the administration of sedation. Encouraging the practice of safe sedation will improve patient safety and help prevent 'never events' from occurring, and will also enhance the patient experience and comfort during the procedure. We 
Table 7 Assorted knowledge on dosage of sedation agentsdata divided by cohorts

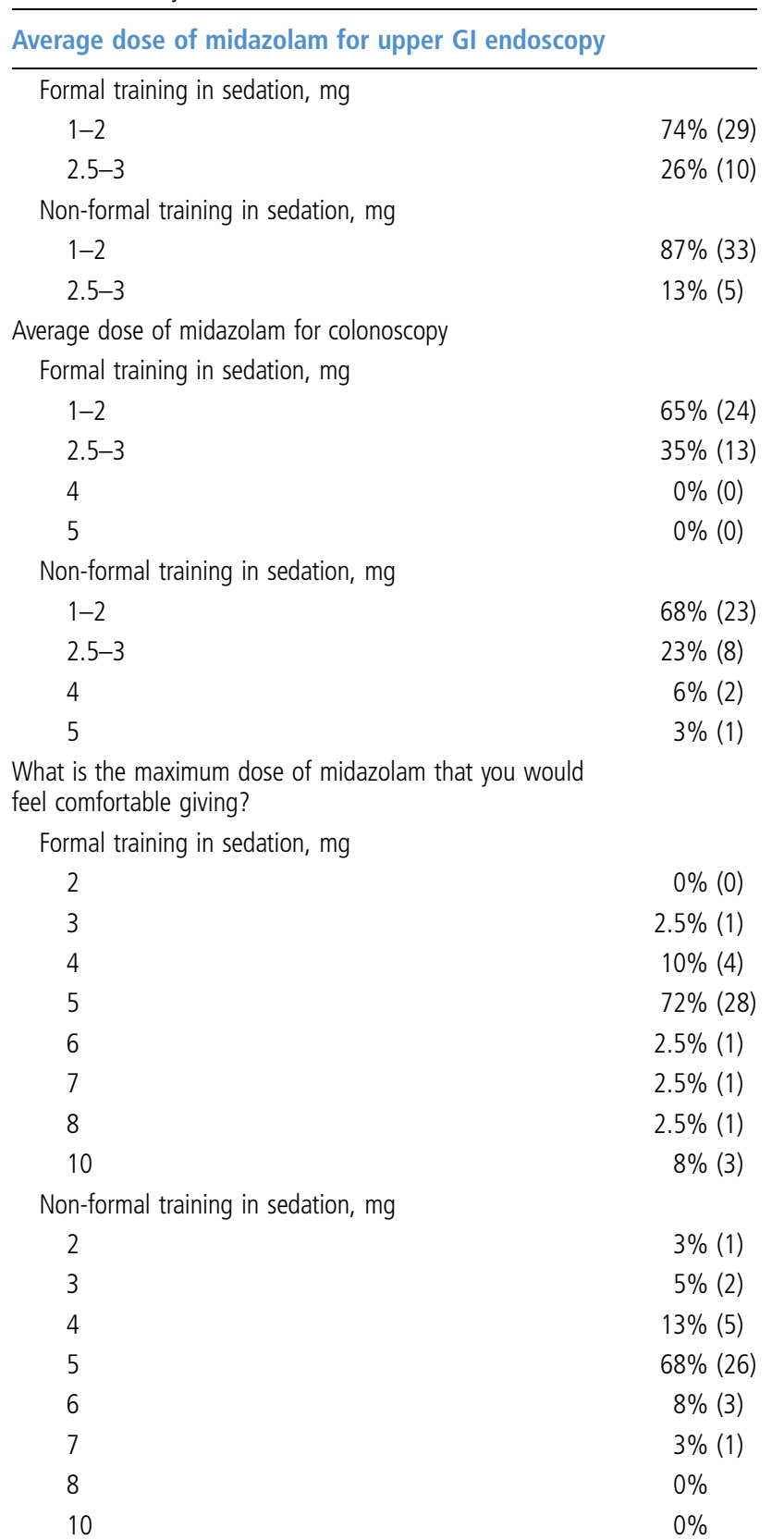

What is the maximum dose of fentanyl that you would feel comfortable giving?

Formal training in sedation, $\mu \mathrm{g}$

\begin{tabular}{lc}
50 & $8 \%(3)$ \\
75 & $11 \%(4)$ \\
80 & $2.5 \%(1)$ \\
100 & $56 \%(20)$ \\
125 & $2.5 \%(1)$ \\
150 & $14 \%(5)$ \\
200 & $6 \%(2)$ \\
Non-formal training in sedation, $\mu g$ & \\
50 & $14 \%(5)$ \\
75 & $8.5 \%(3)$ \\
80 & $68.5 \%(24)$ \\
\hline
\end{tabular}

Continued
Table 7 Continued

Average dose of midazolam for upper GI endoscopy

$\begin{array}{ll}100 & 6 \%(2) \\ 125 & 3 \%(1) \\ 150 & 0 \%(0) \\ 200 & 0 \%(0)\end{array}$

What initial dose of midazolam do you use for an elderly patient undergoing upper $\mathrm{Gl}$ endoscopy?

Formal training in sedation, $\mathrm{mg}$

$\begin{array}{lc}0.5 & 13.5 \%(5) \\ 1 & 67.5 \%(25) \\ 1.5 & 5 \%(2) \\ 2 & 11 \%(4) \\ 2.5 & 3 \%(1) \\ \text { on-formal training in sedation, mg } & \\ 0.5 & 13.5 \%(5) \\ 1 & 73 \%(27) \\ 1.5 & 2.5 \%(1) \\ 2 & 11 \%(4) \\ 2.5 & 0 \%\end{array}$

What initial dose of midazolam do you use for an elderly patient undergoing colonoscopy?

Formal training in sedation, $\mathrm{mg}$

\begin{tabular}{lc}
0.5 & $11 \%(4)$ \\
1 & $55.5 \%(20)$ \\
1.5 & $5.5 \%(2)$ \\
2 & $25 \%(9)$ \\
2.5 & $3 \%(1)$ \\
on-formal training in sedation, mg & \\
0.5 & $12 \%(4)$ \\
1 & $47 \%(16)$ \\
1.5 & $9 \%(3)$ \\
2 & $29 \%(10)$ \\
2.5 & $3 \%(1)$ \\
\hline
\end{tabular}

propose that a formal training session in sedation or an e-learning module (drawing on the expertise from our anaesthetic colleagues) could be incorporated as part of a deanery or trust induction for trainees and regularly reviewed thereafter.

Contributors AM came up with the original idea for the study and helped design the questionnaire and wrote the main article. $\mathrm{KB}$ was involved in designing and distributing the questionnaire to trainees. She also wrote the discussion section of this article. AR helped design the questionnaire and provided the expert knowledge on the common sedation agents used. JG, collated the questionnaire data and performed the descriptive statistical analysis and created the results section. GJ was the senior author, who reviewed the questionnaire and this article prior to its submission.

Competing interests None declared.

Provenance and peer review Not commissioned; externally peer reviewed.

\section{REFERENCES}

1 Dumonceau JM, Riphaus A, Beilenhoff U, et al. European curriculum for sedation training in gastrointestinal endoscopy: 
position statement of the European Society of Gastrointestinal Endoscopy (ESGE) and European Society of Gastroenterology and Endoscopy Nurses and Associates (ESGENA). Endoscopy 2013;45:496-504.

2 Lichtenstein DR, Jagannath S, Baron TH, et al. Standards of Practice Committee of the American Society for Gastrointestinal Endoscopy Guidelines. Sedation and anesthesia in GI endoscopy. Gastrointest Endosc 2008;68:815-26.

3 British Society of Gastroenterology (BSG 2003): Safety and sedation during endoscopic procedures. http://www.bsg.org.uk/ clinical-guidelines/endoscopy/guidelines-on-safety-and-sedationduring-endoscopic-procedures.html

4 NHS Commissioning Board, Serious Incident Framework 2013: An update to the 2010 national framework for reporting and learning from serious incidents requiring investigations. http://www.england.nhs.uk/wp-content/uploads/2013/03/ sif-guide.pdf
5 Ball AJ, Campbell JA, Riley SA. Nitrous oxide use during colonoscopy: a national survey of English screening colonoscopists. Frontline Gastroenterol 2014;5:254-9.

6 Cullinane M, Gray AJG. NCEPOD: scoping our practice. 2004. http://www.ncepod.org.uk/2004report

7 Valori R, Barton R. Joint Advisory Group on GI Endoscopy: BSG quality and safety indicators for endoscopy. 2007. http:// www.thejag.org.uk/downloads\%5CUnit\%20Resources\%5CBSG \%20Quality\%20and\%20Safety\%20Indicators.pdf

8 Joint Advisory Group: Jag Accreditation. https://www. jagaccreditation.org

9 Survey Monkey. http://www.surveymonkey.com

10 Joint Royal College of Physicians Training Board: Specialty Training Curriculum for Gastroenterology. 2010 http://www. jrcptb.org.uk/sites/default/files/2010\%20Gastro\%20 (amendment\%202013).pdf

11 Mohanaruban A, Flanders L, Dor R. Consent in the endoscopy department. Frontline Gastroenterol 2014;5:291-29. 